Piermarco Cannarsa $\cdot$ Yifeng Yu

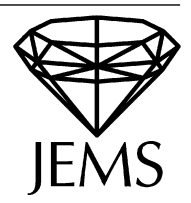

\title{
Singular dynamics for semiconcave functions
}

Received July 15, 2007 and in revised form November 18, 2007

\begin{abstract}
Semiconcave functions are a well-known class of nonsmooth functions that possess deep connections with optimization theory and nonlinear pde's. Their singular sets exhibit interesting structures that we investigate in this paper. First, by an energy method, we analyze the curves along which the singularities of semiconcave solutions to Hamilton-Jacobi equations propagate- the socalled generalized characteristics. This part of the paper improves the main result in [P. Albano, P. Cannarsa, Propagation of singularities for solutions of nonlinear first order partial differential equations, Arch. Ration. Mech. Anal. 162 (2002), 1-23] and simplifies the construction therein. As applications, we recover some known results for gradient flows and conservation laws. Then we derive a simple dynamics for the propagation of singularities of general semiconcave functions. This part of the work is also used to study the singularities of generalized solutions to MongeAmpère equations. We conclude with a global propagation result for the singularities of solutions in weak KAM theory.
\end{abstract}

Keywords. Semiconcave functions, singularities, Hamilton-Jacobi equations, Monge-Ampère equations, weak KAM theory

\section{Introduction}

Semiconcave functions are nonsmooth functions that play an important role in optimal control and partial differential equations. Indeed, the value functions of many optimal control problems, as well as the viscosity solutions of several classes of fully nonlinear pde's, often happen to be either semiconcave or semiconvex (see, for instance, [8]). Therefore, it is interesting to investigate, on the one hand, the structure of the singular set of a general semiconcave function $u$-where a singularity is a point at which $u$ fails to be differentiable. On the other hand, one would rightfully expect extra properties of such a set to hold when $u$ is also a solution to a specific problem, for instance some partial differential equation.

The main purpose of this paper is to construct and study the dynamics that governs the propagation of singularities of a general semiconcave function, as well as to obtain

P. Cannarsa: Dipartimento di Matematica, Università di Roma Tor Vergata, Via della Ricerca Scientifica 1, 00133 Roma, Italy; e-mail: cannarsa@mat.uniroma2.it

Y. Yu: Department of Mathematics, University of Texas at Austin, 1 University Station, C1200 Austin, TX 78712, USA; e-mail: yifengyu@ math.utexas.edu

Mathematics Subject Classification (2000): 26B25, 35A21, 49L25 
new regularity results for singular sets. Major contributions to this analysis were obtained by Albano and the first author in [1], where lower bounds for the Hausdorff dimension of the singular sets of a general semiconcave function were derived. Those results were used, later on, by Rifford [19] and Yu [21] to study the regularity of singular sets. One of the main results in [1] is that, under suitable conditions, singularities propagate along Lipschitz arcs. But does such an arc have any chance of being more regular than just Lipschitz?

In Section 4 of this paper, building on previous work by the second author [20], we use an energy method to select a singular arc which is, in fact, a generalized gradient flow of a semiconcave function. This property is related to classical results by Crandall and Pazy [10] on generalized flows of maximal monotone operators in Hilbert spaces, which have recently been extended to a metric set-up by Ambrosio, Gigli and Savaré [4]. In the much simpler context of Euclidean space, we will reprove such a result in Section 3 by a new method, which is technically very simple and useful to get uniform estimates.

Another interesting result in [1] describes the propagation of singularities along higher dimensional sets. Here, we contribute to the analysis deriving an evolution equation for singular manifolds. We hope that, in the future, this result may help to recover better regularity properties of such sets.

An application of our propagation results to generalized solutions of Monge-Ampère equations is presented in Section 5. This part of the paper is related to previous work [21] by the second author who proved that, when $n=2$, the singularities of a generalized solution propagate along $C^{1}$ curves under some geometrical assumptions which-though necessary in some sense - are in general hard to verify. Theorem 5.1 of this paper, which holds under milder and more natural assumptions than the ones considered in [21], ensures the propagation of singularities along $C^{1}$ curves with the possible exception of a countable set.

When the semiconcave function of interest is a viscosity solutions of a HamiltonJacobi equation, Albano and the first author proved in [2] that singular arcs can be selected as generalized characteristics. In Section 3 of this paper, we use an energy method to study the regularity of a generalized characteristic, and discuss three applications of such a result. The first one is a refined version of the main theorem in [2] (a proof in a similar spirit is given in [20]). The second one, already mentioned above, is that we recover regularity for generalized gradient flows. The third application is that we reprove a result by Dafermos [11] for generalized solutions to 1-d conservation laws. In this last application, our method might not be much simpler than the one in [11], but it shows, in addition, that singularities choose to travel along a path which minimizes some energy.

In the last section, we derive a propagation result for the singularities of solutions in weak KAM theory (see the monograph by Fathi [15] for an introduction to this subject). Although this is no direct application of the theory of Sections 3 and 4 , we think it adds nicely to the paper since it establishes new connections between the propagation of singularities and the global behaviour of Hamiltonian systems.

Outline of the paper. In Section 2, we go over some preliminaries about semiconcave functions and prove a useful approximation lemma. In Section 3, we discuss some properties of generalized characteristics and several applications. In Section 4, we derive the 
dynamics of the propagation of singularities of a general semiconcave function. In Section 5. we give an application to generalized solutions of Monge-Ampère equations. Last, in Section 6 we discuss the propagation of singularities in weak KAM theory.

\section{Preliminaries and approximation lemma}

Throughout this paper, $I_{n}$ is the $n \times n$ identity matrix, and balls are denoted by

$$
B_{r}(x)=\left\{y \in \mathbb{R}^{n}|| y-x \mid<r\right\} \quad \text { and } \quad \bar{B}_{r}(x)=\left\{y \in \mathbb{R}^{n}|| y-x \mid \leq r\right\} .
$$

Moreover, $t \rightarrow s^{+}$is the abbreviation of the fact that $t$ approaches $s$ from the right. Also, $C$ will denote a constant only depending on known quantities.

For any nonempty closed set $S \subset \mathbb{R}^{n}$ we set

$$
\operatorname{dist}(x, S)=\min _{y \in S}|x-y| \quad \forall x \in \mathbb{R}^{n} .
$$

It is well-known that dist $(\cdot, S)$ is continuous and

$$
|\operatorname{dist}(x, S)-\operatorname{dist}(y, S)| \leq|x-y| \quad \forall x, y \in \mathbb{R}^{n} .
$$

Moreover, recalling the definition of the Hausdorff distance of two compact sets $R, S \subset$ $\mathbb{R}^{n}$, that is,

$$
d_{\mathcal{H}}(R, S)=\max \left\{\max _{x \in R} \operatorname{dist}(x, S), \max _{y \in S} \operatorname{dist}(y, R)\right\},
$$

we see that $\operatorname{dist}(x, \cdot)$ has the following continuity property: for all compact sets $R, S \subset \mathbb{R}^{n}$,

$$
|\operatorname{dist}(x, R)-\operatorname{dist}(x, S)| \leq d_{\mathcal{H}}(R, S) \quad \forall x \in \mathbb{R}^{n} .
$$

Let $\Omega$ be a bounded domain (i.e., a connected open subset) of $\mathbb{R}^{n}$. For any function $u: \Omega \rightarrow \mathbb{R}, D u\left(x_{0}\right)=\left(u_{x_{1}}\left(x_{0}\right), \ldots, u_{x_{n}}\left(x_{0}\right)\right)$ denotes the gradient vector of $u$ at $x_{0} \in \Omega$ (if $u$ is differentiable at $\left.x_{0}\right)$, and $D^{2} u\left(x_{0}\right)=\left[u_{x_{i} x_{j}}\left(x_{0}\right)\right]$ the Hessian matrix of $u$ at $x_{0}$ (if $u$ is twice differentiable at $x_{0}$ ).

In the following, $u$ will stand for a (linearly) semiconcave function on $\Omega$, for which we assume that there are constants $L_{i}>0, i=0,1,2$, such that

$$
\begin{cases}\text { (a) }|u(x)| \leq L_{0} & \text { for all } x \in \Omega, \\ \text { (b) }|D u(x)| \leq L_{1} & \text { for a.e. } x \in \Omega, \\ \text { (c) } x \mapsto u(x)-\frac{L_{2}}{2}|x|^{2} & \text { is concave in } \Omega .\end{cases}
$$

We recall that property (c) above, which is one of the possible definitions of linear semiconcavity, implies that

$$
D^{2} u \leq L_{2} I_{n}
$$

whenever $u$ happens to be twice differentiable in $\Omega$ (see, e.g., [8, Proposition 1.1.3])

\footnotetext{
1 As a matter of fact, one can show that any linearly semiconcave function is twice differentiable a.e. and [2.5] is satisfied for a.e. $x \in \Omega$ (see, e.g., [8, Theorem 2.3.1]) and [14, Chapter 6.3]).
} 
The superdifferential of $u$ at $x_{0}$ is defined by

$$
D^{+} u\left(x_{0}\right)=\left\{p \in \mathbb{R}^{n} \mid u(x) \leq u\left(x_{0}\right)+p \cdot\left(x-x_{0}\right)+o\left(\left|x-x_{0}\right|\right)\right\},
$$

whereas

$$
\begin{aligned}
D^{*} u\left(x_{0}\right)=\left\{p \in \mathbb{R}^{n} \mid \text { there exists a sequence } x_{m} \rightarrow x_{0} \text { as } m \rightarrow \infty\right. \\
\text { such that } \left.\operatorname{Du}\left(x_{m}\right) \text { exists and } p=\lim _{m \rightarrow \infty} D u\left(x_{m}\right)\right\}
\end{aligned}
$$

is the set of all limiting (or achievable) gradients of $u$ at $x_{0}$.

It is well known that, for a semiconcave function $u, D^{+} u\left(x_{0}\right)$ is a compact convex set and

$$
D^{+} u\left(x_{0}\right)=\operatorname{co} D^{*} u\left(x_{0}\right),
$$

where, for each subset $K \subset \mathbb{R}^{n}$, co $K$ stands for the convex hull of $K$, i.e., the least closed convex set which contains $K$. Consequently, the superdifferential is nonempty at every point of $\Omega$ and coincides with all the main notions of generalized differentials, such as the proximal superdifferential or Clarke's generalized gradient (see, e.g., [8, Proposition 3.3.6 and 3.6.2]). Moreover, for any $x \in \Omega$, a vector $p \in \mathbb{R}^{n}$ belongs to $D^{+} u(x)$ if and only if

$$
u(y)-u(x)-p \cdot(y-x) \leq \frac{L_{2}}{2}|y-x|^{2}
$$

for any points $x, y \in \Omega$ such that the line segment $[x, y]:=\{\lambda x+(1-\lambda) y \mid \lambda \in[0,1]\}$ is contained in $\Omega$, where $L_{2}$ is the constant in 2.4 (see, e.g., [8, Proposition 3.3.1]). Furthermore, we have, for every $p \in D^{+} u(x)$ and $q \in D^{+} u(y)$,

$$
(q-p) \cdot(y-x) \leq L_{2}|y-x|^{2}
$$

(see, e.g., [8, Proposition 3.3.10]).

The singular set of $u$ is defined to be

$$
\begin{aligned}
\Sigma(u) & =\left\{x \in \Omega \mid u \text { is not differentiable at } x_{0}\right\} \\
& =\left\{x \in \Omega \mid D^{+} u\left(x_{0}\right) \text { contains more than one point }\right\} .
\end{aligned}
$$

Also, for $1 \leq i \leq n$ we set

$$
\Sigma^{i}(u)=\left\{x \in \Sigma(u) \mid \operatorname{dim}\left(D^{+} u\left(x_{0}\right)\right)=i\right\} .
$$

We prepare below a simple approximation lemma which will be frequently used in this work. It is a refinement of Lemma 2.1 in [20].

Lemma 2.1. Let $x_{0} \in \Omega$ and let $V$ be an open subset of $\Omega$ such that

$$
x_{0} \in V \subset \bar{V} \subset \Omega \text {. }
$$

Then for any $p \in D^{+} u\left(x_{0}\right)$ there is a sequence $\left\{u_{m}\right\}_{m \geq 1} \subset C^{\infty}(\bar{V})$ satisfying

(a) $\left|u_{m}(x)\right| \leq L_{0},\left|D u_{m}(x)\right| \leq L_{1}, D^{2} u_{m}(x) \leq L_{2} I_{n}$ for all $x \in V$, 
(b) $\lim _{m \rightarrow \infty} u_{m}=u$ uniformly in $\bar{V}$ and $\lim _{m \rightarrow \infty} D u_{m}\left(x_{0}\right)=p$,

where $L_{i}$ are the constants in 2.4.

Proof. Since $D^{+} u\left(x_{0}\right)=\operatorname{co} D^{*} u\left(x_{0}\right)$, owing to Carathéodory's theorem for all integers $i=1, \ldots, n+1$ there are sequences $\left\{x_{i}^{m}\right\}_{m \geq 1} \subset V$ and $\left\{\lambda_{i}^{m}\right\}_{m \geq 1} \subset[0,1]$ such that, for every $m \geq 1$,

(i) $x_{i}^{m} \in B_{1 / m}\left(x_{0}\right)$ for every $i=1, \ldots, n+1$ and $\sum_{i=1}^{n+1} \lambda_{i}^{m}=1$,

(ii) $D u\left(x_{i}^{m}\right)$ exists for every $i=1, \ldots, n+1$,

(iii) $\left|\sum_{i=1}^{n+1} \lambda_{i}^{m} D u\left(x_{i}^{m}\right)-p\right| \leq 1 / m$.

Since the set-valued map $x \leadsto D^{+} u(x)$ is upper semicontinuous (see, e.g., [8, Proposition 3.3.4]), there are positive numbers $\epsilon_{m}<1 / m$ such that

$$
\underset{x \in \bar{B}_{\epsilon_{m}}\left(x_{i}^{m}\right)}{\operatorname{ess} \sup _{m}} \operatorname{dist}\left(D u\left(x_{i}^{m}\right), D^{+} u(x)\right) \leq m^{-1} \quad \text { for } 1 \leq i \leq n+1 .
$$

Now, fix a function $\eta \in C_{0}^{\infty}\left(B_{1}(0)\right)$ satisfying $\int_{\mathbb{R}^{n}} \eta d x=1$, and define

$$
\eta_{m}(x):=\sum_{i=1}^{n+1} \frac{\lambda_{i}^{m}}{\epsilon_{m}^{n}} \eta\left(\frac{x_{0}-x_{i}^{m}-x}{\epsilon_{m}}\right) \quad \forall x \in \mathbb{R}^{n} .
$$

It is easy to see that

$$
\operatorname{spt}\left(\eta_{m}\right) \subset \bigcup_{i=1}^{n+1} \bar{B}_{\epsilon_{m}}\left(x_{0}-x_{i}^{m}\right) \subset \bar{B}_{2 / m}(0)
$$

where "spt" stands for support, and

$$
\int_{\mathbb{R}^{n}} \eta_{m}(y) d y=1
$$

Let us define, for $m$ sufficiently large,

$$
u_{m}(x):=\int_{\mathbb{R}^{n}} u(x-y) \eta_{m}(y) d y \quad \forall x \in \bar{V} .
$$

It is then immediate to realize that $u_{m}$ satisfies 2.4) and (2.5) with the same constants $L_{i}$ as $u$. Hence, point (a) of the conclusion holds and $\lim _{m \rightarrow \infty} u_{m}=u$ uniformly in $\bar{V}$. So, we only need to verify that $\lim _{m \rightarrow \infty} D u_{m}\left(x_{0}\right)=p$. Since

$$
\begin{aligned}
D u_{m}\left(x_{0}\right) & =\int_{\mathbb{R}^{n}} D u\left(x_{0}-y\right) \eta_{m}(y) d y \\
& =\sum_{i=1}^{n+1} \frac{\lambda_{i}^{m}}{\epsilon_{m}^{n}} \int_{\bar{B}_{\epsilon_{m}}\left(x_{0}-x_{i}^{m}\right)} D u\left(x_{0}-y\right) \eta\left(\frac{x_{0}-x_{i}^{m}-y}{\epsilon_{m}}\right) d y,
\end{aligned}
$$


(2.9) yields

$$
\left|D u_{m}\left(x_{0}\right)-\sum_{i=1}^{n+1} \lambda_{i}^{m} D u\left(x_{i}^{m}\right)\right| \leq \frac{1}{m} .
$$

So, on account of (iii), we conclude that

$$
\left|D u_{m}\left(x_{0}\right)-p\right| \leq\left|D u_{m}\left(x_{0}\right)-\sum_{i=1}^{n+1} \lambda_{i}^{m} D u\left(x_{i}^{m}\right)\right|+\left|\sum_{i=1}^{n+1} \lambda_{i}^{n} D u\left(x_{i}^{m}\right)-p\right| \leq \frac{2}{m} .
$$

Remark 2.2. It is worth noting that the conclusion of Lemma 2.1 entails that, for every $x \in V$, any cluster point of $\left\{D u_{m}(x)\right\}$ belongs to $D^{+} u(x)$. Indeed, let $\left\{D u_{m_{k}}(x)\right\}$ be a subsequence of $\left\{D u_{m}(x)\right\}$ such that $D u_{m_{k}}(x)$ converges to some vector $p_{x} \in \mathbb{R}^{n}$ as $k \rightarrow \infty$. Owing to the bounds in (a),

$$
u_{m_{k}}(y)-u_{m_{k}}(x)-D u_{m_{k}}(x) \cdot(y-x) \leq \frac{L_{2}}{2}|y-x|^{2}
$$

for all $y \in V$ sufficiently close to $x$. Hence, in the limit as $k \rightarrow \infty$,

$$
u(y)-u(x)-p_{x} \cdot(y-x) \leq \frac{L_{2}}{2}|y-x|^{2} .
$$

This ensures that $p_{x} \in D^{+} u(x)$ on account of (2.7).

\section{Generalized characteristics}

Throughout this section we assume that the following conditions hold:

(A1) $u$ is a semiconcave function satisfying (2.4);

(A2) $H=H(p, z, x)$ is a function of class $C^{1}\left(\mathbb{R}^{n} \times \mathbb{R} \times \Omega\right)$ satisfying

(i) $H(\cdot, z, x)$ is convex for each $(z, x) \in \mathbb{R} \times \Omega$;

(ii) for each $(z, x) \in \mathbb{R} \times \Omega$ and $c \in \mathbb{R}$, the $c$-level set $\{p \mid H(p, z, x)=c\}$ does not contain any line segment.

Observe that $u$ and $H$ have no relation to each other. However, in some of the results below, $u$ will be supposed to be a viscosity solution of the equation $H(D u, u, x)=0$ in $\Omega$.

We begin with a definition that is central to our analysis.

Definition 3.1. A Lipschitz continuous arc $\xi:[0, \sigma] \rightarrow \Omega$ is said to be a generalized characteristic for the pair $(u, H)$ if

$$
\dot{\xi}(s) \in \operatorname{co} D_{p} H\left(D^{+} u(\xi(s)), u(\xi(s)), \xi(s)\right) \quad \text { for a.e. } s \in[0, \sigma] .
$$

For a viscosity solution $u$ of $H(D u, u, x)=0$ in $\Omega$, it was proved in [2] that singularities propagate from a given point $x_{0} \in \Sigma(u)$ along a generalized characteristic provided that

$$
0 \notin \operatorname{co} D_{p} H\left(D^{+} u\left(x_{0}\right), u\left(x_{0}\right), x_{0}\right) .
$$

We are interested in the following three questions, related to the above result. 
(Q1) Does 3.1) have a unique solution with given initial data?

(Q2) When do we actually have

$$
\dot{\xi}(s) \in D_{p} H\left(D^{+} u(\xi(s)), u(\xi(s)), \xi(s)\right) \quad \text { for a.e. } s \in[0, \sigma] \text { ? }
$$

(Q3) What kind of regularity can be proved for a generalized characteristic besides Lipschitz continuity?

In the following, we will give partial answers to (Q1)-(Q3) and recover several known interesting results as applications. First, we are going to construct a generalized characteristic $\xi(s)$ such that the right derivative $\dot{\xi}^{+}(0)$ exists. Our strategy is to approximate $u$ with proper smooth functions (similar techniques were used in Yu [15]). Then, for solutions of Hamilton-Jacobi equations, we will show that such an arc consists of singular points under a milder condition than 3.2.

Theorem 3.2. Assume (A1), (A2), let $x_{0} \in \Omega$ and let $p_{0} \in D^{+} u\left(x_{0}\right)$ be such that

$$
H\left(p_{0}, u\left(x_{0}\right), x_{0}\right)=\min _{p \in D^{+} u\left(x_{0}\right)} H\left(p, u\left(x_{0}\right), x_{0}\right) .
$$

Fix a positive number $r<\operatorname{dist}\left(x_{0}, \partial \Omega\right)$ and define

$$
\sigma_{r}=\min \left\{\frac{r}{\left|D_{p} H(p, z, x)\right|+1}\left|x \in \bar{B}_{r}\left(x_{0}\right),\right| z\left|\leq L_{0},\right| p \mid \leq L_{1}\right\} .
$$

Then there is a generalized characteristic $\xi:\left[0, \sigma_{r}\right] \rightarrow \Omega$ for $(u, H)$ starting at $x_{0}$ which satisfies

$$
\begin{aligned}
\dot{\xi}^{+}(0)= & \lim _{s \rightarrow 0^{+}} \frac{\xi(s)-x_{0}}{s}=D_{p} H\left(p_{0}, u\left(x_{0}\right), x_{0}\right), \\
& \lim _{t \rightarrow 0^{+}} \underset{s \in[0, t]}{\operatorname{ess} \sup }\left|\dot{\xi}(s)-\dot{\xi}^{+}(0)\right|=0 .
\end{aligned}
$$

Observe that, in general, a generalized characteristic may well be a constant arc. However, for solutions of $H(D u, u, x)=0$, it was proved in [2] that singularities propagate along genuine shocks (one-to-one generalized characteristics) under assumption (3.2). A first application of Theorem 3.2 ensures that the same conclusion holds under the more natural condition 3.7 below. Indeed, the set $D_{p} H\left(D^{+} u\left(x_{0}\right), u\left(x_{0}\right), x_{0}\right)$ is, in general, smaller than co $D_{p} H\left(D^{+} u\left(x_{0}\right), u\left(x_{0}\right), x_{0}\right)$ (see Example 3.5 below).

Corollary 3.3. Let $u$ be a viscosity solution of $H(D u, u, x)=0$ in $\Omega$. If $x_{0} \in \Sigma(u)$ and

$$
0 \notin D_{p} H\left(D^{+} u\left(x_{0}\right), u\left(x_{0}\right), x_{0}\right)
$$

then, for $r>0$ small enough, there is a generalized characteristic $\xi:\left[0, \sigma_{r}\right] \rightarrow \Omega$ for $(u, H)$ starting from $x_{0}$ such that $\dot{\xi}^{+}(0) \neq 0$ and $\xi(s) \in \Sigma(u)$ for all $\left.s \in\left[0, \sigma_{r}\right]\right|^{2}$

\footnotetext{
$2 \sigma_{r}$ is defined in 3.4 .
} 
The proofs of Theorem 3.2 and Corollary 3.3 have a common part consisting of the following approximation argument. Let $V=B_{r}\left(x_{0}\right)$. Owing to Lemma 2.1, there is a sequence of smooth functions $\left\{u_{m}\right\}$ enjoying properties (a) and (b) in the lemma for $p=p_{0}$. It is easy to see that, for every $m \geq 1$, the Cauchy problem

$$
\left\{\begin{array}{l}
\dot{x}=D_{p} H\left(D u_{m}(x), u_{m}(x), x\right), \\
x(0)=x_{0}
\end{array}\right.
$$

(where we have omitted to specify $s$-dependence) has a $C^{1}$ solution $\xi_{m}:\left[0, \sigma_{r}\right] \rightarrow \Omega$. Moreover, without loss of generality, we can assume that

$$
\lim _{m \rightarrow \infty} \xi_{m}(s)=\xi(s) \quad \text { uniformly in }\left[0, \sigma_{r}\right] .
$$

Hence, a standard argument shows that $\xi(s)$ is a generalized characteristic for $(u, H)$ (see, e.g., [20]).

Proof of Theorem 3.2. Let us prove (3.5) and 3.6. Since $D^{2} u_{m} \leq L_{2} I_{n}$, we obtain by differentiation

$$
\begin{aligned}
\frac{d}{d s} H\left(D u_{m}\left(\xi_{m}(s)\right), u_{m}\left(\xi_{m}(s)\right)\right. & \left., \xi_{m}(s)\right)=H_{p_{i}} H_{p_{j}} u_{m, x_{i} x_{j}}+H_{p_{i}} H_{z} u_{m, x_{i}}+H_{x_{i}} H_{p_{i}} \\
& \leq C\left|D_{p} H\right|^{2}+\left|D_{p} H\right|\left|D u_{m}\right|\left|H_{z}\right|+\left|D_{x} H\right|\left|D_{p} H\right| \leq C,
\end{aligned}
$$

for some constant $C$ depending only on $H, L_{i}$ and $r$. Hence, for all $s \in\left[0, \sigma_{r}\right]$,

$$
H\left(D u_{m}\left(\xi_{m}(s)\right), u_{m}\left(\xi_{m}(s)\right), \xi_{m}(s)\right) \leq H\left(D u_{m}\left(x_{0}\right), u_{m}\left(x_{0}\right), x_{0}\right)+C s .
$$

We claim that

$$
\lim _{m \rightarrow \infty, t \rightarrow 0^{+}} \sup _{s \in[0, t]}\left|D u_{m}\left(\xi_{m}(s)\right)-p_{0}\right|=0 .
$$

Indeed, if this is not the case, then there are sequences $m_{k} \rightarrow \infty$ and $s_{k} \rightarrow 0^{+}$(as $k \rightarrow \infty)$ such that

$$
\lim _{k \rightarrow \infty} D u_{m_{k}}\left(\xi_{m_{k}}\left(s_{k}\right)\right)=p_{1} \neq p_{0} .
$$

It is clear that $p_{1} \in D^{+} u\left(x_{0}\right)$. Owing to 3.10) and 3.3,

$$
H\left(p_{1}, u\left(x_{0}\right), x_{0}\right) \leq H\left(p_{0}, u\left(x_{0}\right), x_{0}\right)=\min _{p \in D^{+} u\left(x_{0}\right)} H\left(p, u\left(x_{0}\right), x_{0}\right) .
$$

Then we must have

$$
H\left(p_{1}, u\left(x_{0}\right), x_{0}\right)=H\left(p_{0}, u\left(x_{0}\right), x_{0}\right)=\min _{p \in D^{+} u\left(x_{0}\right)} H\left(p, u\left(x_{0}\right), x_{0}\right) .
$$

Thus, in view of (A2), $p_{1}=p_{0}$, which is a contradiction. Hence 3.11 holds. Moreover, (3.8) and 3.11 yield

$$
\lim _{m \rightarrow \infty, t \rightarrow 0^{+}} \underset{s \in[0, t]}{\operatorname{ess} \sup }\left|\dot{\xi}_{m}(s)-D_{p} H\left(p_{0}, u\left(x_{0}\right), x_{0}\right)\right|=0 .
$$

By 3.9 and 3.12 we obtain 3.5 and 3.6. 
Proof of Corollary 3.3 Let $\left\{u_{m}\right\}$ and $\left\{\xi_{m}\right\}$ be the above approximating sequences, and let $\xi$ be the generalized characteristic given by Theorem 3.2. Fix $s \in\left[0, \sigma_{r}\right]$ and pass to the limit along a subsequence $m_{k} \rightarrow \infty$. By (3.10) and Remark 2.2 we deduce that, for some $p_{s} \in D^{+} u(\xi(s))$

$$
H\left(p_{s}, u(\xi(s)), \xi(s)\right) \leq H\left(p_{0}, u\left(x_{0}\right), x_{0}\right)+C s .
$$

Observe that $H\left(p_{0}, u\left(x_{0}\right), x_{0}\right)<0$, since $u$ is a solution of $H(D u, u, x)=0$ and $x_{0}$ is a singular point of $u$. Hence, for $r>0$ small enough,

$$
\min _{p \in D^{+} u(\xi(s))} H(p, u(\xi(s)), \xi(s)) \leq \frac{1}{2} H\left(p_{0}, u\left(x_{0}\right), x_{0}\right)<0
$$

for all $s \in\left[0, \sigma_{r}\right]$. Therefore, $\xi\left(\left[0, \sigma_{r}\right]\right) \subset \Sigma(u)$. Finally, the fact that $\dot{\xi}^{+}(0) \neq 0$ is an easy consequence of 3.5 and 3.7.

The following corollary of Theorem 3.2 is a direct consequence of the translation invariance of (3.1).

Corollary 3.4. Suppose that for any $x \in \Omega$ there exists a unique generalized characteristic for $(u, H)$ starting from $x$. Then any generalized characteristic $\xi:[0, \sigma] \rightarrow \Omega$ for $(u, H)$ has right derivative $\dot{\xi}^{+}(s)$ for all $s \in[0, \sigma)$ and

$$
\dot{\xi}^{+}(s)=D_{p} H(p(s), u(\xi(s)), \xi(s)),
$$

where $p(s)$ is the unique point of $D^{+} u(\xi(s))$ such that

$$
H(p(s), u(\xi(s)), \xi(s))=\min _{p \in D^{+} u(\xi(s))} H(p, u(\xi(s)), \xi(s)) .
$$

Moreover, $\dot{\xi}^{+}$is right-continuous.

In general, 3.1 might have more than one solution with given initial data as our next example shows.

Example 3.5. Let $H\left(p_{1}, p_{2}\right)=\frac{1}{4}\left(p_{1}^{4}+p_{2}^{4}\right)-p_{1} / 2-p_{2} / 2$ and choose a concave function $u \in C\left(\mathbb{R}^{2}\right)$ such that

$$
D^{+} u(0,0)=\{(t, 1-t) \mid 0 \leq t \leq 1\},
$$

e.g., $u(x, y)=\min \{x, y\}$. It is easy to check that $(0,0) \in \operatorname{co} D_{p} H\left(D^{+} u(0,0)\right)$. So, the constant $\operatorname{arc} \xi_{0}(s) \equiv(0,0)$ is a (trivial) generalized characteristic for $(u, H)$. However, Theorem 3.2 ensures the existence of another generalized characteristic $\xi_{1}$ for $(u, H)$, starting from $(0,0)$, which satisfies 3.5 . Since

$$
(0,0) \notin D_{p} H\left(D^{+} u(0,0)\right),
$$

we see that $\xi_{0} \neq \xi_{1}$. Hence, there is more than one generalized characteristic passing through $(0,0)$. 
Next, we give several examples where the above uniqueness property holds true.

Example 3.6. For $H(p, x)=\frac{1}{2}|p|^{2}-1$ the corresponding generalized characteristic is the so-called generalized gradient flow. As is well-known for maximal monotone operators (see, e.g., [6] and [5]), the generalized gradient flow with given initial data is unique. Indeed, if $\xi_{1}$ and $\xi_{2}$ are two generalized characteristics for $(u, H)$ starting from $x_{0}$, then $\dot{\xi}_{i}(s) \in D^{+} u\left(\xi_{i}(s)\right)$ for $i=1,2$ and a.e. $s \in[0, \sigma]$. So, 2.8 yields

$$
\frac{1}{2} \frac{d}{d s}\left|\xi_{2}(s)-\xi_{1}(s)\right|^{2}=\left(\dot{\xi}_{2}(s)-\dot{\xi}_{1}(s)\right) \cdot\left(\xi_{2}(s)-\xi_{1}(s)\right) \leq L_{2}\left|\xi_{2}(s)-\xi_{1}(s)\right|^{2} .
$$

Therefore, $\xi_{1} \equiv \xi_{2}$ by Gronwall's lemma.

An immediate consequence of Corollary 3.4 is the following result.

Corollary 3.7. Let $\xi(s):[0, \sigma] \rightarrow \Omega$ be a generalized gradient flow associated with $u$, i.e., a Lipschitz arc satisfying

$$
\dot{\xi}(s) \in D^{+} u(\xi(s)) \quad \text { for a.e. } s \in[0, \sigma] .
$$

Then, for all $s \in[0, \sigma)$,

$$
\dot{\xi}^{+}(s)=p(s) \in D^{+} u(\xi(s)) \quad \text { where } \quad|p(s)|=\min _{p \in D^{+} u(\xi(s))}|p| .
$$

Moreover, $\dot{\xi}^{+}(s)$ is right-continuous.

As a matter of fact, the above property is true in Hilbert spaces (see, e.g., [5, p. 396]), and even in metric spaces (see [4, Theorem 2.4.15]). Clearly, there is a price to pay for greater generality: the more general the set-up, the more technical the proof.

Example 3.8. Let $H(p, x)=p_{n+1}+\left|p^{\prime}\right|^{2}$ where $p:=\left(p^{\prime}, p_{n+1}\right) \in \mathbb{R}^{n} \times \mathbb{R}$. This case corresponds to the Hamilton-Jacobi equation

$$
u_{t}+|D u|^{2}=0
$$

for $u(x, t) \in C\left(\mathbb{R}^{n} \times[0, \infty)\right)$. Adapting the argument of Example 3.6. one can show that the corresponding generalized characteristic is unique with given initial data.

Example 3.9. Let $n=2, p=\left(p_{1}, p_{2}\right) \in \mathbb{R}^{2}$ and $(x, t) \in \mathbb{R} \times[0, \infty)$. Let us consider the Hamiltonian

$$
H(p, x, t)=p_{2}+F\left(p_{1}, x, t\right),
$$

where $F \in C^{2}(\mathbb{R} \times \mathbb{R} \times[0, \infty))$ is a strictly convex function in the $p_{1}$ variable, i.e., $F_{p_{1} p_{1}}>0$. This case corresponds to the Hamilton-Jacobi equation

$$
u_{t}+F\left(u_{x}, x, t\right)=0,
$$

which is, in turn, connected with the conservation law

$$
v_{t}+(F(v, x, t))_{x}=0 .
$$


Indeed, it can be shown (see, e.g., [9]) that $u$ is a viscosity solution of (3.13) if and only if $v=u_{x}$ is an entropy solution of equation (3.14). In [11], Dafermos made a very detailed analysis of the discontinuity points of entropy solutions to 1-d conservation laws, where he proved that the generalized characteristic is unique with given initial data by looking at something called "genuine characteristics". As an application of Corollary 3.4, at least in the homogeneous case, we will recover below Theorem 3.1 of [11] from the point of view of minimizing the energy.

Since $u(\cdot, t)$ is a semiconcave function of $x$, we have, for any $t>0$,

$$
\begin{aligned}
u_{x}^{-}(x, t) & =\lim _{y \rightarrow x^{-}} \frac{u(y, t)-u(x, t)}{y-x}=\max \left\{p_{1} \mid\left(p_{1}, p_{2}\right) \in D^{+} u(x, t)\right\} \\
& =\max \left\{p_{1} \mid\left(p_{1}, p_{2}\right) \in D^{*} u(x, t)\right\}
\end{aligned}
$$

and

$$
\begin{aligned}
u_{x}^{+}(x, t) & =\lim _{y \rightarrow x^{+}} \frac{u(y, t)-u(x, t)}{y-x}=\min \left\{p_{1} \mid\left(p_{1}, p_{2}\right) \in D^{+} u(x, t)\right\} \\
& =\min \left\{p_{1} \mid\left(p_{1}, p_{2}\right) \in D^{*} u(x, t)\right\}
\end{aligned}
$$

(see [8, Theorem 3.3.6]).

Theorem 3.10. Let $n=2$ and

$$
H(p, x, t)=p_{2}+F\left(p_{1}, x, t\right),
$$

where $F \in C^{2}(\mathbb{R} \times \mathbb{R} \times[0, \infty))$ is a strictly convex function in the $p_{1}$ variable. Then (3.1) has a unique solution with given initial data.

Proof. Let $\xi:[0, \sigma] \rightarrow \mathbb{R} \times[0, \infty)$ and $\eta:[0, \sigma] \rightarrow \mathbb{R} \times[0, \infty)$ be two solutions of 3.1) such that $\xi(0)=\eta(0)=\left(x_{0}, t_{0}\right)$. Set $\xi=\left(\xi_{1}, \xi_{2}\right)$ and $\eta=\left(\eta_{1}, \eta_{2}\right)$. Since $F_{p_{1}}$ is increasing with respect to $p_{1}$, for a.e. $s \in[0, \sigma]$ we obtain

$$
\begin{aligned}
& \dot{\xi}(s) \in\left[F_{p_{1}}\left(u_{x}^{+}(\xi(s)), \xi(s)\right), F_{p_{1}}\left(u_{x}^{-}(\xi(s)), \xi(s)\right)\right] \times\{1\}, \\
& \dot{\eta}(s) \in\left[F_{p_{1}}\left(u_{x}^{+}(\eta(s)), \eta(s)\right), F_{p_{1}}\left(u_{x}^{-}(\eta(s)), \eta(s)\right)\right] \times\{1\} .
\end{aligned}
$$

So, $\xi_{2}(s)=\eta_{2}(s)=s+t_{0}$. Moreover, recalling that $F_{p_{1} p_{1}}$ is positive and $F \in C^{2}$, by (2.8) we conclude that

$$
\begin{aligned}
\frac{d}{d s}\left|\xi_{1}(s)-\eta_{1}(s)\right|^{2} & \left(\xi_{1}(s)-\eta_{1}(s)\right)\left(F_{p_{1}}\left(u_{x}\left(\xi_{1}(s), s+t_{0}\right), \xi(s)\right)-F_{p_{1}}\left(u_{x}\left(\eta_{1}(s), s+t_{0}\right), \eta(s)\right)\right) \\
= & \left(\xi_{1}(s)-\eta_{1}(s)\right)\left(\left(F_{p_{1}}\left(u_{x}\left(\xi_{1}(s), s+t_{0}\right), \xi(s)\right)-F_{p_{1}}\left(u_{x}\left(\eta_{1}(s), s+t_{0}\right), \xi(s)\right)\right)\right. \\
& +\left(\xi_{1}(s)-\eta_{1}(s)\right)\left(\left(F_{p_{1}}\left(u_{x}\left(\eta_{1}(s), s+t_{0}\right), \xi(s)\right)-F_{p_{1}}\left(u_{x}\left(\eta_{1}(s), s+t_{0}\right), \eta(s)\right)\right)\right. \\
\leq & C\left|\xi_{1}(s)-\eta_{1}(s)\right|^{2}
\end{aligned}
$$

for a.e. $s \in[0, \sigma]$. Then Gronwall's inequality yields $\xi_{1} \equiv \eta_{1}$. Hence, $\xi \equiv \eta$. 
Now we are going to prove Theorem 3.1 in [11] from the viewpoint of minimizing the energy. As above, we take $n=2$ and

$$
H(p, x, t)=p_{2}+F\left(p_{1}, x, t\right),
$$

where $F \in C^{2}(\mathbb{R} \times \mathbb{R} \times[0, \infty))$ is a strictly convex function in the $p_{1}$ variable.

Theorem 3.11. Let $u$ be a semiconcave solution of the Hamilton-Jacobi equation

$$
u_{t}+F\left(u_{x}, x, t\right)=0 \quad \text { in } \mathbb{R} \times[0, \infty),
$$

and let $\xi:\left[t_{0}, t_{0}+\sigma\right] \rightarrow \mathbb{R} \times[0, \infty)$ be a generalized characteristic for $(u, H)$ starting from a given point $\left(x_{0}, t_{0}\right) \in \mathbb{R} \times[0, \infty)$. Then $\xi(s)=(x(s), s)$ where $\dot{x}^{+}$is rightcontinuous and satisfies, for all $s \in\left[t_{0}, t_{0}+\sigma\right)$,

$$
\dot{x}^{+}(s)= \begin{cases}F_{p_{1}}\left(u_{x}(x(s), s), x(s), s\right) & \text { if }(x(s), s) \notin \Sigma(u), \\ \frac{F\left(u_{x}^{-}(x(s), s), x(s), s\right)-F\left(u_{x}^{+}(x(s), s), x(s), s\right)}{u_{x}^{-}(x(s), s)-u_{x}^{+}(x(s), s)} & \text { if }(x(s), s)) \in \Sigma(u),\end{cases}
$$

Proof. First, observe that, in view of Corollary 3.4 and Theorem $3.10, \dot{\xi}^{+}(s)$ exists for all $s \in\left[t_{0}, t_{0}+\sigma\right)$ and satisfies the characteristic equation

$$
\left\{\begin{array}{l}
\dot{\xi}^{+}(s)=D_{p} H(p(s), \xi(s))=\left(F_{p_{1}}\left(p_{1}(s), \xi(s)\right), 1\right), \\
\xi\left(t_{0}\right)=\left(x_{0}, t_{0}\right),
\end{array}\right.
$$

where $p(s)=\left(p_{1}(s), p_{2}(s)\right) \in \partial D^{+} u(\xi(s))$ is such that

$$
H(p(s), \xi(s))=\min _{p \in D^{+} u(\xi(s))} H(p, \xi(s)),
$$

where the fact that $p(s)$ is on the boundary of $D^{+} u(\xi(s))$ is justified by the absence of critical points of $D_{p} H$. The conclusion easily follows if $\xi(s)=(x(s), s) \notin \Sigma(u)$.

So, suppose $(x(s), s) \in \Sigma(u)$ and observe that for any $(x, t) \in \mathbb{R} \times(0, \infty)$,

$$
D^{*} u(x, t) \subset\left\{\left(p_{1}, p_{2}\right) \in \mathbb{R}^{2} \mid p_{2}+F\left(p_{1}, x, t\right)=0\right\} .
$$

Hence, in view of 3.15 and 3.16 ,

$$
\left\{\begin{array}{l}
p^{+}(s):=\left(u_{x}^{+}(x(s), s),-F\left(u_{x}^{+}(x(s), s), x(s), s\right)\right) \in D^{*} u(x(s), s) \\
p^{-}(s):=\left(u_{x}^{-}(x(s), s),-F\left(u_{x}^{-}(x(s), s), x(s), s\right)\right) \in D^{*} u(x(s), s),
\end{array}\right.
$$

where, thanks to 3.18$), u_{x}^{+}(x(s), s)<u_{x}^{-}(x(s), s)$ since $(x(s), s)$ is a singular point.

We claim that, for all $s \in\left[t_{0}, t_{0}+\sigma\right)$,

$$
p(s) \in] p^{+}(s), p^{-}(s)\left[\subset \partial D^{+} u(x(s), s),\right.
$$

\footnotetext{
3 Actually, $D^{*} u(x, t)=\left\{\left(p_{1}, p_{2}\right) \in D^{+} u(x, t) \mid p_{2}+F\left(p_{1}, x, t\right)=0\right\}$ (see [8. Theorem 6.4.12]).
} 
where $] p, q[$ denotes the open line segment $\{\lambda p+(1-\lambda) q \mid \lambda \in(0,1)\}$. Indeed, for all $p=\left(p_{1}, p_{2}\right) \in D^{*} u(x(s), s)$ there exists $\lambda \in[0,1]$ such that

$$
\left\{\begin{array}{l}
p_{1}=\lambda p_{1}^{+}(s)+(1-\lambda) p_{1}^{-}(s) \\
p_{2} \geq \lambda p_{2}^{+}(s)+(1-\lambda) p_{2}^{-}(s)
\end{array}\right.
$$

where the equality is true by construction whereas the inequality follows from the convexity of $F(\cdot, x, t)$ and 3.18 . Since $D^{+} u(x(s), s)=\operatorname{co} D^{*} u(x(s), s), 3.21$ also holds for all $p \in D^{+} u(x(s), s)$. This shows that $\left[p^{+}(s), p^{-}(s)\right] \subset \partial D^{+} u(x(s), s)$. Moreover, in view of 3.21, for all $p=\left(p_{1}, p_{2}\right) \in D^{+} u(x(s), s)$ we have

$$
\begin{aligned}
H(p, x(s), s) & =p_{2}+F\left(p_{1}, x(s), s\right) \\
& \geq \lambda p_{2}^{+}(s)+(1-\lambda) p_{2}^{-}(s)+F\left(\lambda p_{1}^{+}(s)+(1-\lambda) p_{1}^{-}(s)\right) \\
& =H\left(\lambda p^{+}(s)+(1-\lambda) p^{-}(s), x(s), s\right),
\end{aligned}
$$

which implies, in turn, that $p(s) \in\left[p^{+}(s), p^{-}(s)\right]$. Furthermore, $p(s) \neq p^{ \pm}(s)$ on account of the strict convexity of $F$ with respect to $p_{1}$, which yields, for all $\lambda \in(0,1)$,

$H\left(\lambda p^{+}(s)+(1-\lambda) p^{-}(s), x(s), s\right)<\lambda H\left(p^{+}(s), x(s), s\right)+(1-\lambda) H\left(p^{-}(s), x(s), s\right)$.

Our claim is thus proved.

Finally, use 3.17 to deduce that $\left(\dot{x}^{+}(s), 1\right)$ is a normal vector to the convex set $D^{+} u(x(s), s)$ at $p(s)$. Thus, in view of 3.20 ,

$$
\left.\left(\dot{x}^{+}(s), 1\right) \perp\right] p^{+}(s), p^{-}(s)\left[\quad \forall s \in\left[t_{0}, t_{0}+\sigma\right) .\right.
$$

The conclusion

$$
\left[u_{x}^{-}(x(s), s)-u_{x}^{+}(x(s), s)\right] \dot{x}^{+}(s)=F\left(u_{x}^{-}(x(s), s), x(s), s\right)-F\left(u_{x}^{+}(x(s), s), x(s), s\right)
$$

follows by recalling 3.19 .

Remark 3.12. If $\left(x_{0}, t_{0}\right) \in \Sigma(u)$, then, by Corollary 3.3 and Theorem 3.10 , there exist $\sigma>0$ and a unique Lipschitz arc $\xi:\left[t_{0}, t_{0}+\sigma\right] \rightarrow \Sigma(u)$ (called a shock in [11]) such that

$$
\left\{\begin{array}{l}
\dot{\xi}(s) \in F_{p_{1}}\left(\left[u_{x}^{+}(\xi(s)), u_{x}^{-}(\xi(s))\right], \xi(s)\right) \times\{1\} \quad \text { for a.e. } t \in\left[t_{0}, t_{0}+\sigma\right], \\
\xi\left(t_{0}\right)=\left(x_{0}, t_{0}\right) .
\end{array}\right.
$$

Moreover, owing to Theorem $3.11, \xi(s)=(x(s), s)$ satisfies for all $t \in\left[t_{0}, t_{0}+\sigma\right)$ the so-called Rankine-Hugoniot condition

$$
\dot{x}^{+}(s)=\frac{F\left(u_{x}^{-}(x(s), s), x(s), s\right)-F\left(u_{x}^{+}(x(s), s), x(s), s\right)}{u_{x}^{-}(x(s), s)-u_{x}^{+}(x(s), s)} .
$$

Using the specific structure of two-dimensional topology and the uniqueness of generalized characteristics, one can show that $\sigma=\infty$ (see [11, Theorem 4.2]). 


\section{Singular dynamics for general semiconcave functions}

In this section we will be concerned with the propagation of singularities of general semiconcave functions much in the same spirit as in [1], showing, however, that singularities propagate according to some dynamics. Our work in this section is also motivated by our interest in the singularities of generalized solutions of Monge-Ampère equations.

Let $u$ be a semiconcave function in $\Omega$ satisfying (2.4) and let $x_{0} \in \Omega$. For any $p_{0} \in$ $D^{+} u\left(x_{0}\right)$ we define

$$
N\left(p_{0}\right)=\left\{q \in \mathbb{R}^{n} \mid q \cdot\left(p-p_{0}\right) \geq 0, \forall p \in D^{+} u\left(x_{0}\right)\right\}
$$

and we denote by $N_{p_{0}}$ the normalized elements of $N\left(p_{0}\right)$, that is,

$$
N_{p_{0}}=\left\{q \in N\left(p_{0}\right)|| q \mid=1\right\} .
$$

Notice that $q \in N\left(p_{0}\right)$ iff $-q$ belongs to the normal cone to $D^{+} u\left(x_{0}\right)$ at $p_{0}$.

We now prove a lemma which can be viewed as a differential version of Lemma 4.5 in [1].

Lemma 4.1. Let $x_{0} \in \Omega$ and let $p_{0} \in D^{+} u\left(x_{0}\right)$. Define

$$
\sigma_{0}=\frac{d\left(x_{0}, \partial \Omega\right)}{\max _{q \in N_{p_{0}}}\left|q-p_{0}\right|+L_{1}}
$$

where $L_{1}$ is the constant in (2.4). Then for every $q \in N_{p_{0}}$ there is a unique Lipschitz continuous solution $\xi_{q}(\cdot):\left[0, \sigma_{0}\right] \rightarrow \Omega$ of the initial value problem

$$
\left\{\begin{array}{l}
\dot{\xi}(s) \in q-p_{0}+D^{+} u(\xi(s)) \quad \text { for a.e. } s \in\left[0, \sigma_{0}\right], \\
\xi(0)=x_{0} .
\end{array}\right.
$$

Moreover,

$$
\dot{\xi}_{q}^{+}(0)=q \quad \text { and } \quad \lim _{s \rightarrow 0^{+}} \sup _{q \in N_{p_{0}}}\left|\dot{\xi}_{q}^{+}(s)-q\right|=0 .
$$

Proof. First, we observe that the existence and uniqueness of the solution $\xi_{q}$ of 4.4 is clear: if we let

$$
w(x)=\left(q-p_{0}\right) \cdot x+u(x),
$$

then $\xi_{q}$ is the generalized gradient flow of $w$ introduced in Example 3.6 .

Second, it is immediate that $\xi_{q}(s)$ can be extended up to time $\sigma_{0}$ given by 4.3. and

Third, owing to Corollary $3.7, \dot{\xi}_{q}^{+}(s)$ exists for all $s \in\left[0, \sigma_{0}\right), \dot{\xi}_{q}^{+}$is right-continuous,

$$
\left|\dot{\xi}_{q}^{+}(s)\right|=\min _{p \in q-p_{0}+D^{+} u\left(\xi_{q}(s)\right)}|p| .
$$

Since $q-p_{0}+D^{+} u\left(x_{0}\right)$ is a closed convex set containing $q$, and since

$$
q \cdot\left(p-p_{0}\right) \geq 0 \quad \forall p \in D^{+} u\left(x_{0}\right),
$$


we deduce that $\dot{\xi}_{q}^{+}(0)=q$ and

$$
\lim _{s \rightarrow 0^{+}}\left|\dot{\xi}_{q}^{+}(s)-q\right|=0 .
$$

So, what we really need to prove is that the above limit holds uniformly in $N_{p_{0}}$, i.e., the second assertion in 4.5.

Let $V$ be an open set such that $x_{0} \in V \subset \bar{V} \subset \Omega$, and let $\left\{u_{m}\right\}$ be a sequence of smooth functions with properties (a) and (b) of Lemma 2.1 for $p=p_{0}$. By the same reasoning we used to construct $\xi_{q}$ at the beginning of this proof there is a family of smooth curves $\xi_{m, q}(s):\left[0, \sigma_{1}\right] \rightarrow V$, with $0<\sigma_{1} \leq \sigma_{0}$, such that

$$
\left\{\begin{array}{l}
\dot{\xi}_{m, q}(s)=q-p_{0}+D u_{m}\left(\xi_{m, q}(s)\right), \quad s \in\left[0, \sigma_{1}\right], \\
\xi_{m, q}(0)=x_{0} .
\end{array}\right.
$$

Also, by a standard continuous dependence argument,

$$
\lim _{m \rightarrow \infty} \sup _{q \in N_{p_{0}}}\left|\xi_{m, q}-\xi_{q}\right|=0 \quad \text { uniformly in }\left[0, \sigma_{1}\right] .
$$

Moreover, since $\left|D u_{m}\right| \leq L_{1}$ in $V,\left\{\dot{\xi}_{m, q}\right\}$ is bounded in $L^{2}\left(0, \sigma_{1} ; \mathbb{R}^{n}\right)$. So,

$$
\dot{\xi}_{m, q} \rightarrow \dot{\xi}_{q} \quad \text { in } L^{2}\left(0, \sigma_{1} ; \mathbb{R}^{n}\right) .
$$

Let us introduce the quadratic Hamiltonian

$$
H(p):=q \cdot\left(p-p_{0}\right)+\frac{1}{2}\left|p-p_{0}\right|^{2} .
$$

Then $\dot{\xi}_{m, q}(s)=D_{p} H\left(D u_{m}\left(\xi_{m, q}(s)\right)\right)$. Since $D^{2} u_{m} \leq L_{2} I_{n}$, for every $s \in\left[0, \sigma_{1}\right]$,

$$
\begin{aligned}
\frac{d}{d s} H\left(D u_{m}\left(\xi_{m, q}(s)\right)\right) & =D_{p} H\left(D u_{m}\left(\xi_{m, q}(s)\right)\right) \cdot D^{2} u_{m}\left(\xi_{m, q}(s)\right) D_{p} H\left(D u_{m}\left(\xi_{m, q}(s)\right)\right) \\
& \leq L_{2}\left|D_{p} H\left(D u_{m}\left(\xi_{m, q}(s)\right)\right)\right|^{2} \leq C,
\end{aligned}
$$

whence

$$
\begin{aligned}
H\left(D u_{m}\left(\xi_{m, q}(s)\right)\right) & \leq H\left(D u_{m}\left(x_{0}\right)\right)+C s \\
& =q \cdot\left(D u_{m}\left(x_{0}\right)-p_{0}\right)+\frac{1}{2}\left|D u_{m}\left(x_{0}\right)-p_{0}\right|^{2}+C s .
\end{aligned}
$$

Therefore, for every $\epsilon>0$ there is a number $0<s_{\epsilon} \leq \sigma_{1}$ and an integer $m_{\epsilon} \geq 1$ such that

$$
\left.q \cdot\left[D u_{m}\left(\xi_{m, q}(s)\right)-p_{0}\right]+\frac{1}{2} \mid D u_{m}\left(\xi_{m, q}(s)\right)\right)-\left.p_{0}\right|^{2}<\epsilon
$$

for all $s \in\left[0, s_{\epsilon}\right]$, all $m \geq m_{\epsilon}$ and all $q \in N_{p_{0}}$. Now, after possibly reducing $s_{\epsilon}$ and enlarging $m_{\epsilon}$, a compactness argument yields

$$
\min _{q \in N_{p_{0}}} q \cdot\left[D u_{m}\left(\xi_{m, q}(s)\right)-p_{0}\right] \geq-\epsilon
$$


for all $s \in\left[0, s_{\epsilon}\right]$ and all $m \geq m_{\epsilon}$. Thus, by (4.8),

$$
\sup _{q \in N_{p_{0}}}\left|\dot{\xi}_{m, q}(s)-q\right|=\sup _{q \in N_{p_{0}}}\left|D u_{m}\left(\xi_{m, q}(s)\right)-p_{0}\right| \leq 2 \sqrt{\epsilon}
$$

for all $s \in\left[0, s_{\epsilon}\right]$ and all $m \geq m_{\epsilon}$. Therefore, for $h>0$ sufficiently small,

$$
\sup _{q \in N_{p_{0}}} \frac{1}{h} \int_{s}^{s+h}\left[\dot{\xi}_{m, q}(t)-q\right] \cdot\left[\dot{\xi}_{q}(t)-q\right] d t \leq C \sqrt{\epsilon}
$$

for all $s \in\left[0, s_{\epsilon}\right)$ and all $m \geq m_{\epsilon}$. Thus, recalling (4.7),

$$
\sup _{q \in N_{p_{0}}} \frac{1}{h} \int_{s}^{s+h}\left|\dot{\xi}_{q}(t)-q\right|^{2} d t \leq C \sqrt{\epsilon}
$$

Since $\dot{\xi}_{q}^{+}(t)$ is continuous from the right, in the limit as $h \downarrow 0$,

$$
\sup _{q \in N_{p_{0}}}\left|\dot{\xi}_{q}^{+}(s)-q\right|^{2} \leq C \sqrt{\epsilon}
$$

for a.e. $s \in\left[0, s_{\epsilon}\right)$. So 4.5 holds.

We now state the main result of this section.

Theorem 4.2. Suppose $x_{0} \in \Sigma(u)$ and $p_{0} \in \partial D^{+} u\left(x_{0}\right) \backslash D^{*} u\left(x_{0}\right) \neq \emptyset$. Then there is a number $\tau>0$ and a Lipschitz continuous map $f:[0, \tau] \times N_{p_{0}} \rightarrow \Sigma(u)$ with the following properties:

(a) for all $q \in N_{p_{0}}, f(\cdot, q)$ solves the generalized characteristic problem

$$
\left\{\begin{array}{l}
\partial_{s} f(s, q) \in q-p_{0}+D^{+} u(f(s, q)) \quad \text { for a.e. } s \in[0, \tau], \\
f(0, q)=x_{0}
\end{array}\right.
$$

(b) $\partial_{s}^{+} f(0, q)=q$ and

$$
\lim _{s \rightarrow 0^{+}} \sup _{q \in N_{p_{0}}}\left|\partial_{s}^{+} f(s, q)-q\right|=0
$$

where, for $s \in[0, \tau)$,

$$
\partial_{s}^{+} f(s, q):=\lim _{t \rightarrow s^{+}} \frac{f(t, q)-f(s, q)}{t-s}
$$

(c) we have 4

$$
\inf _{[0, \tau] \times N_{p_{0}}} \operatorname{diam} D^{+} u(f(s, q))>0
$$

\footnotetext{
${ }^{4}$ Here, for each $K \subset \mathbb{R}^{n}$, we have set $\operatorname{diam} K=\sup \{|x-y| \mid x, y \in K\}$.
} 
(d) we have

$$
\liminf _{r \rightarrow 0^{+}} r^{-v} \mathcal{H}^{v}\left(f\left([0, \tau] \times N_{p_{0}}\right) \cap B_{r}\left(x_{0}\right)\right)>0
$$

where $v=\operatorname{dim} N\left(p_{0}\right)$ and $\mathcal{H}^{v}$ stands for Hausdorff measure..$^{5}$

The following is a direct consequence of Theorem 4.2. It will be used in Section 5 where we study the singularities of generalized solutions of Monge-Ampère equations in two dimensions.

Corollary 4.3. Suppose $x_{0} \in \Sigma(u)$ and $p_{0} \in \partial D^{+} u\left(x_{0}\right) \backslash D^{*} u\left(x_{0}\right) \neq \emptyset$. Choose $q \in$ $\mathbb{R}^{n} \backslash 0$ such that

$$
q \cdot\left(p-p_{0}\right) \geq 0 .
$$

Then there is a number $\tau>0$ and a Lipschitz continuous curve $\xi:[0, \tau] \rightarrow \Sigma(u)$ with the following properties:
(a) $\dot{\xi}(s) \in q-p_{0}+D^{+} u(\xi(s))$ for a.e. $s \in[0, \tau]$;
(b) $\dot{\xi}^{+}(0)=q$;
(c) $\lim _{s \rightarrow 0^{+}}\left|\dot{\xi}^{+}(s)-q\right|=0$;
(d) $\inf _{[0, \tau]} \operatorname{diam} D^{+} u(\xi(s))>0$.

Proof of Theorem 4.2. Let $f(s, q):=\xi_{q}(s)$ for $(s, q) \in\left[0, \sigma_{0}\right] \times N_{p_{0}}$, where $\xi_{q}$ and $\sigma_{0}$ are from Lemma 4.1 To complete the proof, we only need to show that

(i) $f$ is Lipschitz continuous;

(ii) there exists $\tau \in\left(0, \sigma_{0}\right]$ such that (c) holds;

(iii) property (4.11) holds.

Proof of $(i)$. By construction, for every $q \in N_{p_{0}}$ and a.e. $s \in\left[0, \sigma_{0}\right]$ we have

$$
\left|\partial_{s}^{+} f(s, q)\right| \leq \max _{q \in N_{p_{0}}}\left|q-p_{0}\right|+L_{1} .
$$

So, $f$ is Lipschitz continuous in the $s$ variable, uniformly in $q$. Thus, we only need to prove that $f$ is Lipschitz continuous in the $q$ variable, with Lipschitz constant independent of $s$. In other terms, setting

$$
w(s)=\left|f\left(s, q_{1}\right)-f\left(s, q_{2}\right)\right|^{2}=\left|\xi_{q_{1}}(s)-\xi_{q_{2}}(s)\right|^{2} \quad \text { for } 0 \leq s \leq \sigma_{0},
$$

our goal is to show that

$$
w(s) \leq C\left|q_{1}-q_{2}\right|^{2} \quad \text { for } 0 \leq s \leq \sigma_{0} .
$$

Since $w(s)$ is Lipschitz continuous, a straight calculation shows that for a.e. $s \in\left[0, \sigma_{0}\right]$,

$$
\frac{d}{d s} w(s)=\left(\xi_{q_{1}}(s)-\xi_{q_{2}}(s)\right) \cdot\left(q_{1}-q_{2}\right)+\left(p_{1}(s)-p_{2}(s)\right) \cdot\left(\xi_{q_{1}}(s)-\xi_{q_{2}}(s)\right),
$$

\footnotetext{
5 Notice that $\operatorname{dim} N\left(p_{0}\right)=1+\operatorname{dim}_{\mathcal{H}} N_{p_{0}}$, where $\operatorname{dim}_{\mathcal{H}}$ stands for Hausdorff dimension.
} 
where $p_{i}(s) \in D^{+} u\left(\xi_{q_{i}}(s)\right)$ for $i=1,2$. By Cauchy's inequality and 2.8),

$$
\frac{d}{d t} w(s) \leq \sqrt{w(s)}\left|q_{1}-q_{2}\right|+L_{2} w(s) \quad \text { for a.e. } s \in\left[0, \sigma_{0}\right] .
$$

Then, $v(s):=e^{-L_{2} s} w(s)$ satisfies

$$
\frac{d}{d s} v(s) \leq C \sqrt{v(s)}\left|q_{1}-q_{2}\right| \quad \text { for a.e. } s \in\left[0, \sigma_{0}\right] .
$$

Since $v(0)=0$, we derive

$$
v(s) \leq C\left|q_{1}-q_{2}\right|^{2} .
$$

So (4.14) holds.

Proof of (ii). We will argue by contradiction. If (ii) does not hold, there are sequences of positive numbers $\left\{s_{m}\right\}_{m \geq 1}$ and vectors $\left\{q_{m}\right\} \subset N_{p_{0}}$ such that $s_{m} \rightarrow 0$ as $m \rightarrow \infty$ and

$$
\lim _{m \rightarrow \infty} \operatorname{diam} D^{+} u\left(f\left(s_{m}, q_{m}\right)\right)=0 .
$$

Since

$$
\partial_{s}^{+} f(s, q) \in q-p_{0}+D^{+} u(f(s, q)),
$$

recalling 4.10 , we find that

$$
\lim _{m \rightarrow \infty} \min _{p \in D^{+} u\left(f\left(s_{m}, q_{m}\right)\right)}\left|p-p_{0}\right|=0 .
$$

Combining this with 4.15 , we get

$$
\lim _{m \rightarrow \infty} \max _{p \in D^{+} u\left(f\left(s_{m}, q_{m}\right)\right)}\left|p-p_{0}\right|=0 .
$$

For each $m \in \mathbb{N}$, choose $p_{m} \in D^{*} u\left(f\left(s_{m}, q_{m}\right)\right)$. Then we have

$$
\lim _{m \rightarrow \infty} p_{m}=p_{0}
$$

So, $p_{0} \in D^{*} u\left(x_{0}\right)$ contrary to the assumption. Note that (c) implies that $f\left([0, \tau] \times N_{p_{0}}\right) \subset$ $\Sigma(u)$.

Proof of (iii). We will refine the proof of [1, Theorem 5.2]. Let us denote by $L\left(p_{0}\right)$ the linear subspace of $\mathbb{R}^{n}$ generated by $N\left(p_{0}\right)$, and by $\pi_{v}: \mathbb{R}^{n} \rightarrow L\left(p_{0}\right)$ the orthogonal projection. Now, recall the translation invariance of Hausdorff's measure and observe that

$$
\mathcal{H}^{\nu}\left(\left[f\left([0, \tau] \times N_{p_{0}}\right)-x_{0}\right] \cap B_{r}(0)\right) \geq \mathcal{H}^{v}\left(\pi_{\nu}\left(\left[f\left([0, \tau] \times N_{p_{0}}\right)-x_{0}\right] \cap B_{r}(0)\right)\right)
$$

to conclude that the inequality

$$
\liminf _{r \rightarrow 0^{+}} r^{-v} \mathcal{H}^{v}\left(\pi_{v}\left(\left[f\left([0, \tau] \times N_{p_{0}}\right)-x_{0}\right] \cap B_{r}(0)\right)\right)>0
$$


suffices to obtain the conclusion. Then set

$$
F(s, q):=\pi_{\nu}\left(f(s, q)-x_{0}\right), \quad(s, q) \in[0, \tau] \times N_{p_{0}},
$$

and use 4.13, once again to derive

$$
F\left([0, r] \times N_{p_{0}}\right) \subset F\left([0, \tau] \times N_{p_{0}}\right) \cap B_{r M}(0),
$$

where $M=\max _{q \in N_{p_{0}}}\left|q-p_{0}\right|+L_{1}$, and eventually deduce that 4.16 follows from

$$
\liminf _{r \rightarrow 0^{+}} r^{-v} \mathcal{H}^{v}\left(F\left([0, r] \times N_{p_{0}}\right)\right)>0 .
$$

Next, to show 4.17) set, for any $r>0$,

$$
N\left(p_{0}, r\right)=\left\{q \in N\left(p_{0}\right)|| q \mid \leq r\right\} .
$$

Observe that

$$
F\left([0, r] \times N_{p_{0}}\right)=\phi\left(N\left(p_{0}, r\right)\right)
$$

where $\phi: N\left(p_{0}, r\right) \rightarrow \mathbb{R}^{n}$ is the continuous map defined by

$$
\phi(q)= \begin{cases}F(|q|, q /|q|) & \text { if } q \neq 0, \\ 0 & \text { if } q=0 .\end{cases}
$$

Owing to (b), $\rho(r):=\max _{q \in N\left(p_{0}, r\right)}|\phi(x)-x|$ satisfies

$$
\frac{\rho(r)}{r}=\max _{q \in N\left(p_{0}, r\right)} \frac{1}{r} \pi_{\nu}\left(\int_{0}^{|q|}\left[\partial_{s} f\left(s, \frac{q}{|q|}\right)-\frac{q}{|q|}\right] d s\right) \rightarrow 0 \quad \text { as } r \rightarrow 0^{+} .
$$

Hence, by Lemma 4.4 below, $\phi\left(N\left(p_{0}, r\right)\right) \supset \operatorname{int}_{\rho(r)}\left(N\left(p_{0}, r\right)\right)$. Thus, by 4.18], it suffices to show that

$$
\liminf _{r \rightarrow 0^{+}} r^{-v} \mathcal{H}^{v}\left(\operatorname{int}_{\rho(r)}\left(N\left(p_{0}, r\right)\right)\right)>0 .
$$

On the other hand, for some $\alpha>0$ and all $r>0$,

$$
\frac{\mathcal{H}^{v}\left(\operatorname{int}_{\rho(r)}\left(N\left(p_{0}, r\right)\right)\right)}{\mathcal{H}^{v}\left(N\left(p_{0}, r\right)\right)}=\left(1-\alpha \frac{\rho(r)}{r}\right)^{v} \rightarrow 1 \quad \text { as } r \rightarrow 0^{+}
$$

thanks to 4.19. So, 4.20 holds true and the proof is complete.

Lemma 4.4. Let $\Lambda \subset \mathbb{R}^{n}$ be a domain such that $0 \in \bar{\Lambda}$, and let $\phi: \bar{\Lambda} \rightarrow \mathbb{R}^{n}$ be a continuous map such that, for some $\rho>0$,

$$
|\phi(x)-x| \leq \rho \quad \forall x \in \bar{\Lambda} .
$$

Then

$$
\phi(\bar{\Lambda}) \supset \operatorname{int}_{\rho}(\Lambda):=\{x \in \Lambda \mid \operatorname{dist}(x, \partial \Lambda) \geq \rho\} .
$$


Proof. If $\operatorname{int}_{\rho}(\Lambda)=\emptyset$, then the conclusion is trivial. Otherwise, let $y \in \operatorname{int}_{\rho}(\Lambda)$ and observe that $y=\phi\left(x_{y}\right)$ for some $x_{y} \in \bar{B}_{\rho}(y)$ iff $x_{y}$ is a fixed point of the map

$$
\psi: \bar{B}_{\rho}(y) \rightarrow \mathbb{R}^{n}, \quad \psi(x)=y+x-\phi(x) .
$$

Now, in view of 4.21), $|\psi(x)-y| \leq \rho$ for every $x \in \bar{B}_{\rho}(y)$, or $\psi\left(\bar{B}_{\rho}(y)\right) \subset \bar{B}_{\rho}(y)$. Then the conclusion follows from Brouwer's fixed point theorem.

Remark 4.5. Theorem 4.2 says that, under suitable assumptions, the singularities of $u$ propagate along Lipschitz continuous surfaces $\left\{f\left(\{s\} \times N_{p_{0}}\right)\right\}_{s \in[0, \tau]}$. Obviously, the Hausdorff dimension of $f\left(\{s\} \times N_{p_{0}}\right)$ is bounded above by $\operatorname{dim}_{\mathcal{H}} N_{p_{0}}$. We are interested in the following two questions.

Q1. Is it true that, when $s>0$ is sufficiently small, the Hausdorff dimension of $f(\{s\} \times$ $N_{p_{0}}$ ) is equal to $\operatorname{dim}_{\mathcal{H}} N_{p_{0}}$ ?

Q2. What else can we say about the regularity of $\left\{f\left(\{s\} \times N_{p_{0}}\right)\right\}$ when $s$ is sufficiently small?

We will investigate these two questions in the future.

\section{Application to Monge-Ampère equations}

Let $X$ and $Y$ be two bounded open subsets of $\mathbb{R}^{n}$ with the same Lebesgue measure. In this section we assume that $u: X \rightarrow \mathbb{R}$ is a convex function such that

(A1) $\phi_{u}=D u$ maps $X$ onto $Y$ (in the a.e. sense);

(A2) the Monge-Ampère equation det $D^{2} u=1$ is satisfied in the sense that

$$
\int_{X} \eta(D u) d x=\int_{Y} \eta(y) d y
$$

for any continuous function $\eta: \bar{Y} \rightarrow \mathbb{R}$.

Then we know that $\phi_{u}$ is an optimal transfer map from $X$ to $Y$ with quadratic cost, i.e., $\phi_{u}$ minimizes the functional

$$
\int_{X}|x-\phi(x)|^{2} d x
$$

among all the volume preserving maps $\phi$ from $X$ to $Y$ (see, e.g., [13]). If $Y$ is convex, then $u$ is smooth by a regularity result due to Caffarelli [7]. If $Y$ is a smooth domain but not convex, then the singular set $\Sigma(u)$ might be nonempty even if $X$ is a ball (see, for instance, Example 5.3 below). It is, therefore, an interesting problem to derive more information about $\Sigma(u)$.

For $n=2$, the second author proved in [21] that $\Sigma(u) \backslash \overline{\Sigma^{2}(u)}$ is a $C^{1}$ manifold, provided that $X$ is convex and $Y$ satisfies suitable geometric assumptions. Consequently, if $\Sigma^{2}(u)$ is empty, then $\Sigma(u)$ is a $C^{1}$ manifold, that is, the singularities of $u$ propagate along $C^{1}$ curves. However, in general, we cannot expect $\Sigma^{2}(u)$ to be empty except for some very special cases. This is one of our motivations to find the dynamics of the propagation of singularities for semiconcave functions. 
The following result, which removes part of the geometric assumptions made in [21], is an application of Corollary 4.3 Let us recall that $\left[p_{1}, p_{2}\right]$ denotes the line segment joining two points $p_{1}, p_{2} \in \mathbb{R}^{n}$, and, for any $x \in X$, the subdifferential of $u$ at $x$ is the convex set $D^{-} u(x)=-D^{+}(-u)(x)$. For convex functions-which $u$ is assumed to be in this section- $D^{-} u(x)$ coincides with the classical subdifferential of convex analysis.

Theorem 5.1. Let $X$ and $Y$ be two bounded domains of $\mathbb{R}^{2}$ with the same Lebesgue measure, let $X$ be convex, and assume that, for any compact convex set $K \subset \mathbb{R}^{2}$,

$$
K \cap Y=\emptyset \Rightarrow \partial K \not \subset \partial Y .
$$

Let $u: X \rightarrow \mathbb{R}$ be a convex function with properties (A1), (A2), and let $x_{0} \in \Sigma(u)$.

(i) Then $\partial D^{-} u\left(x_{0}\right) \backslash \bar{Y} \neq \emptyset$ and, for any $p_{0} \in \partial D^{-} u\left(x_{0}\right) \backslash \bar{Y}$ and $q \in \mathbb{R}^{2} \backslash\{0\}$ such that

$$
q \cdot\left(p-p_{0}\right) \leq 0 \quad \forall p \in D^{-} u\left(x_{0}\right),
$$

there is a Lipschitz arc $\xi:[0, \sigma] \rightarrow \Sigma(u)$ satisfying

$$
\left\{\begin{array}{l}
\dot{\xi}(s) \in p_{0}-q-D^{-} u(\xi(s)) \quad \text { for a.e. } s \geq 0, \\
\xi(0)=x_{0}
\end{array}\right.
$$

and

$$
\inf _{s \in[0, \sigma]} \operatorname{diam} D^{-} u(\xi(s))>0 .
$$

(ii) If, for any $p_{1}, p_{2} \in \partial Y$,

$$
\left[p_{1}, p_{2}\right] \cap Y=\emptyset \Rightarrow\left[p_{1}, p_{2}\right] \cap \partial Y=\left\{p_{1}, p_{2}\right\},
$$

then $\dot{\xi}$ is continuous in $[0, \sigma] \backslash \Gamma$, where $\Gamma$ is the countable set $[0, \sigma] \cap \xi^{-1}\left(\Sigma^{2}(u)\right)$.

Proof. First, let us recall that, according to [7], there is a function $v \in C^{\infty}(Y)$ such that $D v(Y)$ is a dense open subset of $X$. Also, $u \in C^{\infty}(D v(Y))$ and $D u(D v(y))=y$ for all $y \in Y$.

(i) Let $x_{0} \in \Sigma(u)$. From the above considerations it follows that $D^{*} u\left(x_{0}\right) \subset \bar{Y}$. We claim that

$$
D^{-} u\left(x_{0}\right) \cap Y=\emptyset \text {. }
$$

Indeed, suppose there is a vector $p \in D^{-} u\left(x_{0}\right) \cap Y$. Then $x_{0} \neq x_{p}:=D v(p)$ since $u$ is differentiable at $x_{p}$, and $p=D u\left(x_{p}\right)$. Hence, being convex, $u$ must be linear along the line segment connecting $x_{0}$ and $x_{p}$. On the other hand, routine arguments based on (5.1) show that this is impossible when $n=2$. We have thus reached a contradiction, which shows (5.5).

Now, 5.5 and assumption (5.2) imply that $\partial D^{-} u\left(x_{0}\right) \backslash \bar{Y} \neq \emptyset$. Moreover, owing to the inclusion $D^{*} u\left(x_{0}\right) \subset \bar{Y}$ and $\left.\sqrt{5.5}\right), D^{*} u\left(x_{0}\right) \subset \partial Y$. Therefore,

$$
p_{0} \in \partial D^{-} u\left(x_{0}\right) \backslash \bar{Y} \Rightarrow p_{0} \in \partial D^{-} u\left(x_{0}\right) \backslash D^{*} u\left(x_{0}\right) \text {. }
$$

So, the conclusion of (i) follows from Corollary 4.3 applied to $-u$. 
(ii) To begin, observe that $\xi([0, \sigma]) \cap \Sigma^{2}(u)$ is (at most) countable (see, e.g., Corollary 4.1.13 in [8]). Also, since $\dot{\xi}^{+}(0)=q \neq 0, \xi(s)$ is one-to-one when $s$ is small enough. Hence, $\Gamma=[0, \sigma] \cap \xi^{-1}\left(\Sigma^{2}(u)\right)$ is countable. Now, let $s_{0} \in[0, \sigma] \backslash \Gamma$, so that $\xi\left(s_{0}\right) \in \Sigma^{1}(u) \backslash \Sigma^{2}(u)$. We claim that

$$
\lim _{s \rightarrow s_{0}}\left(p_{0}-q-D^{-} u(\xi(s))\right)=p_{0}-q-D^{-} u\left(\xi\left(s_{0}\right)\right) .
$$

Indeed, $\lim \sup _{s \rightarrow s_{o}} D^{*} u(\xi(s)) \subset D^{*} u\left(\xi\left(s_{0}\right)\right)$. So, owing to [5.4), $D^{*} u\left(\xi\left(s_{0}\right)\right)$ consists of exactly two points. Moreover, on account of 5.3), $D^{*} u(\xi(s))$ cannot collapse to a point as $s \rightarrow s_{0}$. Therefore, $D^{*} u(\xi(s)) \rightarrow D^{*} u\left(\xi\left(s_{0}\right)\right)$ as $s \rightarrow s_{0}$, and also $D^{-} u(\xi(s)) \rightarrow$ $D^{-} u\left(\xi\left(s_{0}\right)\right)$. Our claim is thus proved.

Recalling that, by Corollary 3.7 .

$$
\left|\dot{\xi}^{+}(s)\right|=\min _{p \in D^{-} u(\xi(s))}\left|p_{0}-q-p\right|
$$

and applying 5.6 , we conclude that $\dot{\xi}^{+}(s) \rightarrow \dot{\xi}^{+}\left(s_{0}\right)$ as $s \rightarrow s_{0}$. Since $\xi$ is differentiable almost everywhere in $[0, \sigma]$, this says that $\dot{\xi}\left(s_{0}\right)$ exists. We have thus shown that $\dot{\xi}$ exists and is continuous at all points of $[0, \sigma] \backslash \Gamma$.

Remark 5.2. The above constructive proof may give an insight into the role played by assumption (5.2). Indeed, the analysis of Section 4-in fact, the main result of [1]pointed out that the basic property that forces the singularity at $x_{0}$ to propagate is that $\partial D^{-} u\left(x_{0}\right) \backslash D^{*} u\left(x_{0}\right) \neq \emptyset$. Since $D u$ is an optimal transfer plan from $X$ to $Y$, it is natural that the geometry of $Y$ may cause such a property to hold true. On the other hand, simple examples like the one below show $D u$ may well have isolated singularities when (5.2) is violated. As for the stronger condition (5.4), again we see from the proof how it essentially entails the continuity of $D^{-} u$ along the shock.

Example 5.3. Let $r>0$ and define the open sets (with the same Lebesgue measure)

$$
X=\left\{x \in \mathbb{R}^{2}|| x \mid<1\right\}, \quad Y=\left\{\left.x \in \mathbb{R}^{2}\left|r^{2}<\right| x\right|^{2}<1+r^{2}\right\} .
$$

Then it is easy to check that the convex function $u(x):=f(|x|)$, where

$$
f(t)=\int_{0}^{t} \sqrt{r^{2}+s^{2}} d s, \quad t \geq 0,
$$

gives an optimal transfer map from $X$ to $Y$ with an isolated singularity at the origin.

The higher dimensional case is much harder to analyze. The only results we have, for the moment, hold under very restrictive conditions on the structure of $\Sigma(u)$. This is why, in this paper, we have only discussed the case of $n=2$. 


\section{Propagation of singularities in weak KAM theory}

In this section, we show that the singularities of solutions in weak KAM theory propagate in some sense under appropriate conditions. For convenience, we focus on the Hamiltonian $H(p, x)=\frac{1}{2}|p|^{2}+V(x)$, where $V(x)$ is a smooth $\mathbb{T}^{n}$-periodic function and $\mathbb{T}^{n}$ is the $n$-dimensional flat torus. One of the basic facts of weak KAM theory is that, for each $P \in \mathbb{R}^{n}$, there is a unique real number, denoted $\bar{H}(P)$, such that the so-called cell problem

$$
\frac{1}{2}|P+D v(x)|^{2}+V(x)=\bar{H}(P), \quad x \in \mathbb{R}^{n},
$$

has a $\mathbb{T}^{n}$-periodic, Lipschitz continuous solution $v$ (see, for instance, [18], [12] and [15]). Here, we assume $v$ to solve 6.1 in the sense of viscosity solutions. The above function $\bar{H}: \mathbb{R}^{n} \rightarrow \mathbb{R}$, called the effective (or averaged) Hamiltonian, is a convex function satisfying

$$
\min _{\mathbb{R}^{n}} \bar{H}=\bar{H}(0)=\max _{\mathbb{T}^{n}} V
$$

Let $v$ be a viscosity solution of (6.1). According to [17, Theorem 3.3], $v$ is semiconcave. Since $v$ is a global solution, it is interesting to see how the global dynamics affects the singular set of $v$. For this purpose, we give the following result showing that the singularities of $v$ propagate, in some sense, if $\bar{H}(P)>\max _{\mathbb{T}^{n}} V$. We denote by $S^{n-1}$ the $(n-1)$-dimensional sphere $\left\{x \in \mathbb{R}^{n}|| x \mid=1\right\}$.

Theorem 6.1. Let $v$ be a $\mathbb{T}^{n}$-periodic semiconcave solution of 6.1 and suppose

$$
\bar{H}(P)>\max _{\mathbb{T}^{n}} V .
$$

Let $x_{0} \in \Sigma(v)$ and let $\Omega$ be an arbitrary bounded open set containing $x_{0}$ such that $\partial \Omega$ is homeomorphic to $S^{n-1}$. Then

$$
\partial \Omega \cap \Sigma(v) \neq \emptyset .
$$

Proof. We will argue by contradiction and suppose that $v$ is differentiable on $\partial \Omega$. Let $u(x):=P \cdot x+v(x)$ and observe that $u$ is also semiconcave and has the same singular set as $v$. Then known results in the calculus of variations (see [15, Section 4.5]; see also [8. Theorem 4.6.9]) ensure that, for any differentiability point $x$ of $u$, there is a unique smooth curve $\xi_{x}:(-\infty, 0] \rightarrow \mathbb{R}^{n}$ such that $u$ is differentiable at $\xi_{x}(t)$ for all $t \leq 0$ and

$$
\left\{\begin{array}{l}
\dot{\xi}_{x}(t)=D u(\xi(t))=P+D v(\xi(t)) \quad(t \leq 0), \\
\xi_{x}(0)=x
\end{array}\right.
$$

Note that $\xi_{x}$ is an absolute minimizing curve, i.e., for any $T<0$ and any Lipschitz arc $\xi:[T, 0] \rightarrow \mathbb{T}^{n}$ satisfying $\xi(0)=x_{0}$ and $\xi(T)=\xi_{x}(T)$, we have

$$
\mathcal{L}_{\mathcal{T}}\left(\xi_{x}\right) \leq \mathcal{L}_{\mathcal{T}}(\xi)
$$

where

$$
\mathcal{L}_{\mathcal{T}}(\xi)=\int_{T}^{0}\left[\frac{1}{2}|\dot{\xi}|^{2}-V(\xi)\right] d t
$$


Hence, $\xi_{x}$ satisfies the Hamiltonian system

$$
\left\{\begin{array} { l } 
{ \dot { \xi } = \eta } \\
{ \dot { \eta } = - D V ( \xi ) }
\end{array} \quad \text { with initial conditions } \quad \left\{\begin{array}{l}
\xi(0)=x \\
\eta(0)=D u(x) .
\end{array}\right.\right.
$$

Also, since $u$ is semiconcave, $x \leadsto D^{+} u(x)$ is upper semicontinuous. Consequently, for every $x \in \mathbb{T}^{n} \backslash \Sigma(u)$, we have

$$
\lim _{\Sigma(u) \not y \rightarrow x} D u(y)=D u(x) .
$$

In particular, this holds for every $x \in \partial \Omega$. So, owing to the continuous dependence on $x$ of the solution of (6.5), we conclude that $x \mapsto \xi_{x}(t)$ is continuous on $\partial \Omega$ for every $t \leq 0$.

Now, for any $t<0$ define the map $\gamma_{t}: \partial \Omega \rightarrow \mathbb{R}^{n}$ by

$$
\gamma_{t}(x)=\xi_{x}(t) \quad \forall x \in \partial \Omega .
$$

In view of the above considerations, $\gamma_{t}$ is continuous. Moreover, $\gamma_{t}$ is one-to-one thanks to the forward uniqueness property of the (generalized) gradient flow recalled in Example 3.6 Therefore, $\gamma_{t}$ is a homeomorphism of $\partial \Omega$ onto the set $\Gamma_{t}:=\gamma_{t}(\partial \Omega)$. Consequently, $\Gamma_{t}$ is homeomorphic to $S^{n-1}$.

Moreover, $t \mapsto \Gamma_{t}$ is continuous in the sense that, for some constant $C \geq 0$,

$$
d_{\mathcal{H}}\left(\Gamma_{s}, \Gamma_{t}\right) \leq C|s-t| \quad \forall s, t \geq 0,
$$

where $d_{\mathcal{H}}$ denotes the Hausdorff distance. Indeed, let $y \in \Gamma_{s}$. Then $y=\xi_{x}(s)$ for some $x \in \partial \Omega$. So, in view of (6.4),

$$
\operatorname{dist}\left(y, \Gamma_{t}\right) \leq\left|\xi_{x}(s)-\xi_{x}(t)\right| \leq\|D u\|_{\infty}|s-t| .
$$

Hence, $\max _{y \in \Gamma_{s}} \operatorname{dist}\left(y, \Gamma_{t}\right) \leq\|D u\|_{\infty}|s-t|$. This yields 6.6 since the remaining term of definition (2.2) can be similarly bounded.

Next, owing to 6.4, for every $x \in \partial \Omega$ and $t \leq 0$ we have

$$
\begin{aligned}
P \cdot\left(x-\xi_{x}(t)\right)+v(x)-v\left(\xi_{x}(t)\right) & =u(x)-u\left(\xi_{x}(t)\right)=\int_{t}^{0} D u\left(\xi_{x}(s)\right) \cdot \dot{\xi}_{x}(s) d s \\
& =2 \int_{t}^{0}\left[\bar{H}(P)-V\left(\xi_{x}(s)\right)\right] d s \\
& \geq-2 t\left[\bar{H}(P)-\max _{\mathbb{T}^{n}} V\right] .
\end{aligned}
$$

Since $v$ is bounded (being $\mathbb{T}^{n}$-periodic) and $\bar{H}(P)>\max _{\mathbb{T}^{n}} V$, we must have

$$
P \cdot \xi_{x}(T)<P \cdot x_{0}-1 \quad \forall x \in \partial \Omega
$$

for all $T<0$ sufficiently large in absolute value. Hereafter, $T<0$ will be a fixed number such that

$$
\Gamma_{T} \subset\left\{y \in \mathbb{R}^{n} \mid P \cdot y<P \cdot x_{0}-1\right\} .
$$


Now, the Jordan-Brouwer theorem (see, e.g., [16, Corollary 18.7]) ensures that, for any $t \leq 0, \mathbb{R}^{n} \backslash \Gamma_{t}$ has two connected components both with boundary $\Gamma_{t}$, one of whichlabelled $\Omega_{t}$-is bounded. Observe that, for every $t \geq 0$,

$$
\operatorname{dist}\left(x, \bar{\Omega}_{t}\right) \leq \operatorname{dist}\left(x, \Gamma_{t}\right) \quad \forall x \in \mathbb{R}^{n} .
$$

Therefore,

$$
\max _{x \in \bar{\Omega}_{s}} \operatorname{dist}\left(x, \bar{\Omega}_{t}\right) \leq \max _{x \in \bar{\Omega}_{s}} \operatorname{dist}\left(x, \Gamma_{t}\right)=\max _{x \in \Gamma_{s}} \operatorname{dist}\left(x, \Gamma_{t}\right) .
$$

Hence, owing to 6.6,

$$
d_{\mathcal{H}}\left(\bar{\Omega}_{s}, \bar{\Omega}_{t}\right) \leq d_{\mathcal{H}}\left(\Gamma_{s}, \Gamma_{t}\right) \leq C|s-t| \quad \forall s, t \geq 0 .
$$

The above considerations allow us to use the signed distance from $\Gamma_{t}$ defined by

$$
d_{\Gamma_{t}}(x)= \begin{cases}\operatorname{dist}\left(x, \Gamma_{t}\right) & \text { if } x \in \bar{\Omega}_{t}, \\ -\operatorname{dist}\left(x, \Gamma_{t}\right) & \text { if } x \in \bar{\Omega}_{t}^{c} .\end{cases}
$$

Clearly, $x \mapsto d_{\Gamma_{t}}(x)$ is continuous. We claim that, for every $x \in \mathbb{R}^{n}, t \mapsto d_{\Gamma_{t}}(x)$ is also continuous. Indeed, let $\bar{t} \geq 0$ be fixed. If $x \in \Gamma_{\bar{t}}$, then (6.6) yields

$$
\left|d_{\Gamma_{t}}(x)-d_{\Gamma_{\bar{t}}}(x)\right|=\operatorname{dist}\left(x, \Gamma_{t}\right) \leq d_{\mathcal{H}}\left(\Gamma_{t}, \Gamma_{\bar{t}}\right) \leq C|t-\bar{t}| \quad \forall t \geq 0 .
$$

Suppose $x \in \Omega_{\bar{t}}$ and let $\delta=\operatorname{dist}\left(x, \Gamma_{\bar{t}}\right)$. Owing to 2.3 and (6.6,

$$
\left|\operatorname{dist}\left(x, \Gamma_{t}\right)-\operatorname{dist}\left(x, \Gamma_{\bar{t}}\right)\right| \leq C|t-\bar{t}| .
$$

Therefore, $\operatorname{dist}\left(x, \Gamma_{t}\right)>\delta / 2$ for $|t-\bar{t}|<\delta /(2 C)$. Should $x$ belong to $\bar{\Omega}_{t}^{c}$ for some $t$ satisfying $|t-\bar{t}|<\delta /(2 C)$, we would have $d_{\mathcal{H}}\left(\bar{\Omega}_{t}, \bar{\Omega}_{\bar{t}}\right)>\delta / 2$ contrary to 6.8). Thus, $x \in \bar{\Omega}_{t}$ for all $|t-\bar{t}|<\delta /(2 C)$. So,

$$
\left|d_{\Gamma_{t}}(x)-d_{\Gamma_{\bar{t}}}(x)\right|=\left|\operatorname{dist}\left(x, \Gamma_{t}\right)-\operatorname{dist}\left(x, \Gamma_{\bar{t}}\right)\right| \leq C|t-\bar{t}| .
$$

Finally, if $x \in \Omega_{\bar{t}}^{c}$, then (6.8) ensures that $x \in \Omega_{t}^{c}$ for every $t$ such that $|t-\bar{t}|<\delta /(2 C)$. This yields 6.9) once again and proves the claimed continuity of $t \mapsto d_{\Gamma_{t}}(x)$.

To complete the proof, define

$$
h(t)=d_{\Gamma_{t}}\left(x_{0}\right) \quad \forall t \leq 0 .
$$

Then $h$ is a continuous function such that $h(0)>0$, since $x_{0} \in \Omega$, and $h(T)<0$, on account of 6.7$)$. Therefore, $h\left(t_{0}\right)=0$ for some $t_{0} \in(T, 0)$. So, $x_{0} \in \Gamma_{t_{0}}$, contradicting the fact that $v$ is differentiable on $\Gamma_{t}$ for all $t \leq 0$.

Remark 6.2. It is not clear to us whether singularities actually propagate along a continuous curve up to infinity. We will investigate this problem in the future.

Acknowledgments. The authors are grateful to the referees for their useful comments and suggestions.

The first author was supported in part by the Italian PRIN 2005 program "Metodi di viscosità, metrici e di teoria del controllo in equazioni alle derivate parziali nonlineari", and the second author by the NSF grant D0601403. 


\section{References}

[1] Albano, P., Cannarsa, P.: Structural properties of singularities of semiconcave functions. Ann. Scuola Norm. Sup. Pisa Cl. Sci. (4) 28, 719-740 (1999) Zbl 0957.26002 MR 1760538

[2] Albano, P., Cannarsa, P.: Propagation of singularities for solutions of nonlinear first order partial differential equations. Arch. Ration. Mech. Anal. 162, 1-23 (2002) Zbl 1043.35052 MR 1892229

[3] Ambrosio, L., Cannarsa, P., Soner, H. M.: On the propagation of singularities of semi-convex functions. Ann. Scuola Norm. Sup. Pisa Cl. Sci. (4) 20, 597-616 (1993) Zbl 0874.49041 MR 1267601

[4] Ambrosio, L., Gigli, N., Savaré, G.: Gradient Flows in Metric Spaces and in the Space of Probability Measures. Lectures in Math. ETH Zürich, Birkhäuser, Basel (2005) Zbl 1090.35002 MR 2305058

[5] Aubin, J.-P., Ekeland, I.: Applied Nonlinear Analysis. Wiley, New York (1984) Zbl 1115.47049 MR 0749753

[6] Brézis, H.: Opérateurs maximaux monotones et semi-groupes de contractions dans les espaces de Hilbert. North-Holland, Amsterdam (1973) Zbl 0252.47055 MR 0348562

[7] Caffarelli, L. A.: The regularity of mappings with a convex potential. J. Amer. Math. Soc. 5, 99-104 (1992) Zbl 0753.35031 MR 1124980

[8] Cannarsa, P., Sinestrari, C.: Semiconcave Functions, Hamilton-Jacobi Equations, and Optimal Control. Progr. Nonlinear Differential Equations Appl. 58, Birkhäuser, Boston (2004) Zbl 1095.49003 MR 2041617

[9] Caselles, V.: Scalar conservation laws and Hamilton-Jacobi equations in one space variable. Nonlinear Anal. 18, 461-469 (1992) Zbl 0755.35067 MR 1152721

[10] Crandall, M. G., Pazy, A.: Semi-groups of nonlinear contractions and dissipative sets. J. Funct. Anal. 3, 376-418 (1969) Zbl 0182.18903 MR 0243383

[11] Dafermos, C. M.: Generalized characteristics and the structure of solutions of hyperbolic conservation laws. Indiana Univ. Math. J. 26, 1097-1119 (1977) Zbl 0377.35051 MR 0457947

[12] Evans, L. C.: Periodic homogenization of certain fully nonlinear PDE. Proc. Roy. Soc. Edinburgh 120, 245-265 (1992) Zbl 0457947 MR 1159184

[13] Evans, L. C.: Partial differential equations and Monge-Kantorovich mass transfer. In: Current Developments in Mathematics, 1997, S. T. Yau (ed.), Int. Press, 26-79 (1999). Latest version: math.berkeley.edu/ evans/Monge-Kantorovich.survey.pdf Zbl 0954.35011 MR 1698853

[14] Evans, L. C., Gariepy, R. F.: Measure Theory and Fine Properties of Functions. Stud. Adv. Math., CRC Press, Ann Arbor (1992) Zbl 0804.28001 MR 1158660

[15] Fathi, A.: The Weak KAM Theorem in Lagrangian Dynamics. Cambridge Stud. Adv. Math., to appear

[16] Greenberg, M. J.: Lectures on Algebraic Topology. W. A. Benjamin, New York-Amsterdam (1967) Zbl 0169.54403 MR 0215295

[17] Lions, P.-L.: Generalized Solutions of Hamilton-Jacobi Equations. Res. Notes in Math. 69, Pitman, Boston (1982) Zbl 0497.35001 MR 0667669

[18] Lions, P.-L., Papanicolaou, G., Varadhan, S. R. S.: Homogenization of Hamilton-Jacobi equations. Unpublished (ca. 1988)

[19] Rifford, L.: Singularities of viscosity solutions and thestabilization problem in the plane. Indiana Univ. Math. J. 52, 1373-1395 (2003) Zbl 1119.93058 MR 2010731

[20] Yu, Y.: A simple proof of the propagation of singularities for solutions of Hamilton-Jacobi equations. Ann. Scuola Norm. Sup. Pisa Cl. Sci. (5) 5, 439-444 (2006) Zbl pre05211204 MR 2297718

[21] Yu, Y.: Singular set of a convex potential in two dimensions. Comm. Partial Differential Equations 32, 1883-1894 (2007) Zbl 1138.35019 MR 2372491 